\title{
Writing together to foster wellbeing: Doctoral writing groups as spaces of wellbeing
}

Beasy $^{\mathrm{a} 1}$, Kim; S. Emery ${ }^{\mathrm{a}}$, L. Dyer ${ }^{\mathrm{b}}$; B. Coleman ${ }^{\mathrm{a}}$; D. Bywaters ${ }^{\mathrm{c}}$; T. Garrad ${ }^{\text {d. J. }}$ Crawford $^{\text {e }}$ K. Swarts ${ }^{\text {fe }}$; S. Jahangiri ${ }^{\mathrm{g}}$

${ }^{a}$ Faculty of Education, University of Tasmania, Launceston, Australia; ${ }^{b}$ College of Sciences and Engineering, University of Tasmania, Launceston, Australia; 'School of Nursing,, College of Health and Medicine, University of Tasmania, Launceston, Australia; ${ }^{d}$ Faculty of Humanities, Arts, Social Sciences and Education, ${ }^{d}$ University of New England, Armidale, Australia; ${ }^{e}$ Tasmanian Institute of Learning and Teaching, ${ }^{f}$ Tasmanian School of Business and Economics, University of Tasmania, Launceston, Australia; ${ }^{g}$ National Centre for Marine Engineering and Hydrodynamics, University of Tasmania, Launceston, Australia

1 *CONTACT. Dr Kim Beasy. Email: Kim.Beasy@utas.edu.au 


\begin{abstract}
Candidate wellbeing is recognised as a continual challenge for doctoral programs, with government mandates requiring an institutional response. This paper explores the experiences of candidates undertaking intensive writing sessions ('Write-Ins') and their influence on their wellbeing. Exploratory findings demonstrate opportunities for Write-In models to contribute positively to 'Spaces of Wellbeing'. Spaces of Wellbeing theory (Fleuret \& Atkinson, 2007) highlights four dimensions of space that influence wellbeing: capability, security, integrative and therapeutic spaces. Findings show the Write-Ins contributed positively to wellbeing by offering space for candidates to enhance writing productivity, to work to their own pace, to connect with others, and to work flexibly.
\end{abstract}

Keywords: doctoral candidate; spaces of wellbeing; postgraduate wellbeing; doctoral writing.

\title{
Introduction
}

The wellbeing of doctoral students is attracting increased attention in the research literature and University discourses internationally. The World Health Organisation (WHO) encompasses wellbeing in its definition of health, indicating that health is "a state of complete physical, mental and social wellbeing and not merely the absence of disease or infirmity" (WHO, 2014). Our university, like many others globally, seeks effective responses to growing concerns about the mental health and wellbeing of postgraduate students (Higher Education Standards Panel - HESP, 2017). The Australian Higher Education Standards Framework highlights a requirement for universities to respond to student safety and wellbeing and portrays a genuine commitment to the promotion and achievement of wellbeing among diverse cohorts (Tertiary Education Quality and Standards Agency - TEQSA, 2018). Yet, concerns about doctoral candidate wellbeing continue, with 25 percent of university 
postgraduates experiencing mental health issues (Evans, Bira, Gastelum, Weiss, \& Vanderford, 2018).

A bleak picture is painted of the existing mental health conditions of postgraduate research students, with increased rates of suicide and suicidal ideation reported compared to undergraduates (Silverman, Meyer, Sloane, Raffel, \& Pratt, 1997), and similar increases in depression, anxiety and stress (Bazrafkan, Shokrpour, Yousefi, \& Yamani, 2016). Conditions of candidature that can negatively impact on doctoral candidates' mental health include social isolation, challenging relationships with research supervisors and managing parenting, caring and financial commitments (Crawford \& Probert, 2017). We contend that this "mental health crisis in graduate education" (Evans et al., 2018) should be a matter of deep concern for universities, but responses need to extend beyond individuals to the deeply ingrained ideas about how doctoral studies are conceived.

Doctoral studies are situated in a broader education landscape subject to contemporary neoliberal dynamics that are "fundamentally restructuring what education does, how it is controlled, and who benefits from it throughout the world" (Apple, 2009, p. 1). The contexts that enable and support postgraduate study are impacted by the consequences of changes brought about by global competition and free-market forces (Baylis, Smith \& Owens, 2017). While this is altering traditional contexts of doctoral studies, for example, by tightening timeframes for completion (Sampson \& Comer, 2010), the expectations of 'deep' engagement remain in terms of the requirement for doctoral students to contribute to the production and reproduction of discipline-specific knowledge (ibid).

The adherence of universities to a free-market model has had consequences for the way that higher education and research is conceived and performed. In sum, 
doctoral candidates are faced with an array of personal and professional stressors throughout their candidature. While a whole-of-institution response is oftern suggested (HESP, 2017), there is a need for opportunities to deeply evaluate efforts to address postgraduate wellbeing.

Research has revealed numerous approaches universities have adopted for supporting doctoral student wellbeing. This includes offering mindfulness meditation for students (Barry et al., 2018), providing access to non-academic support such as learning and physical disability support, counselling and career advice (Waight \& Giordano, 2018), and fostering peer support initiatives (Verlie et al. 2017). In addition, a growing body of literature finds a positive relationship between the formation of writing groups and the wellbeing of doctoral candidates. Verlie et al. (2017) describe the establishment of doctoral networks with the purpose of staging writing retreats that offer opportunities to connect with like-minded people and develop the confidence to upskill as writers. Other studies have found additional social and emotional benefits to stem from participating in writing groups, including feeling a sense of community with other doctoral candidates (Maher et al., 2008), and a lessening of the angst associated with often-solitary doctoral writing (Mewburn, Osborne \& Caldwell, 2014).

Writing groups represent opportunities to cultivate periods of time expressly set aside to undertake thesis writing. A recent Australian study of the experiences of doctoral candidates determined that many struggle to find time for writing amongst work commitments and family and caring obligations (Crawford \& Probert, 2017). Importantly, writing groups provide doctoral candidates with positive writing experiences free of critique and judgement (Mewburn et al., 2014) and can contribute to the construction of an academic identity (Cuthbert, Spark \& Burke, 2009; Wilmot \& 
McKenna, 2018). However, few studies have investigated the attributes of writing groups that enable positive wellbeing to occur.

In this paper, we examine how a writing group initiative developed by doctoral candidates to support their research and mental wellbeing realised these aspirations. We begin this by explaining on our context and introducing the intensive writing group initiative at the centre of this study (the 'Write-In') and present the method for evaluating the writing sessions. This research was guided by the research question:

How does a Write-In program act as, and contribute to, spaces of wellbeing for doctoral students?

\section{The ‘Write-In' Program}

The Write-In program was conducted over a period of six months duration in 2018. Write-In sessions were held in a large university board room at regular intervals (usually fortnightly) on a Saturday between 9am and 5pm. Financial resources obtained through a student union grant covered the cost of lunch for participants taken at a cafe close to the writing venue. The authorship team consists of individuals who led, participated in, or sought funding for the Write-In sessions (see method for bias minimisation notes).

The board room was a 'dignified space' - quiet, well lit and with high quality furniture - in which candidates could work quietly on their thesis. An adjacent staff kitchen and lounge space could be used for conversations or phone calls. During the winter months, the board room was a warm and comfortable space. Multiple space configurations were possible in the boardroom; some candidates worked at the large central table, others worked at small tables around the edge of the room or on one of the two couches in the room. 
Candidates were invited to participate in the Write-Ins through bulk emails sent to all doctoral candidates studying at the University. A total of ten sessions were conducted over the six months. The sessions were self-directed with minimal facilitation, with diverse disciplines represented, including business, architecture, education and nursing among others. What emerged from the program was a core group of doctoral candidates participating fortnightly, and a group of periodic attendees (e.g. those with competing interests including work and family commitments).

The average number of participants at a session was ten and over the ten sessions, candidates collectively worked for 651 hours. Using insights from interviews, we conservatively estimate that candidates wrote a collective 93,000 words over the ten sessions or 1,200 words per session, per person. In our exploration of the influence Write-Ins had on candidate wellbeing we found that generating writing was only one of many benefits.

\section{Spaces of Wellbeing}

Spaces of wellbeing theory (SoWT; Fleuret \& Atkinson, 2007) originates from the field of geography and aims to broaden understandings of spatial, social, and environmental aspects of health and their consequences for the wellbeing of populations. SoWT is underpinned by the theories of needs (Diener \& Lucas, 2000), relative standards theory (Schyns, 1998), and the capabilities approach (Nussbaum, 2001). SoWT considers spaces not only in terms of the physical qualities of spaces and facilities but also their social and relational qualities. The four dimensions of the SoWT model comprise spaces of capability, integrative spaces, therapeutic spaces, and spaces of security (Fleuret $\&$ Atkinson, 2007). 
The four dimensions of the SoWT model are subsequently explained and were used to consider the ways that Write-Ins enable wellbeing through spatial and relational processes, similar to previous studies adopting SoWT (e.g. Kilpatrick, Emery, Adler \& Farmer, 2019; Woodgate et al., 2017). We consider the SoWT framework a particularly relevant theoretical lens for analysing the data in this study in response to recent concerns of poor doctoral candidate wellbeing (Emery et al., 2018; Crawford \& Probert, 2017; Evans et al., 2018).

\section{Spaces of Capability}

Spaces of capability focuses on the self-fulfilment of what is 'made capable' or actualised within the space. The components under consideration in this dimension relate to the nature of the work being produced. For the writing sessions, this was related to each student's production of writing associated with their doctorate, and associated practices (e.g. literature readings). Capability draws upon Sen’s understanding of capability as relevant in the assessment of wellbeing, based on the idea of individuals having the freedom and agency to achieve their valued outcomes (Nussbaum, 2001).

\section{Integrative Spaces}

Integrative spaces emphasise the relationships or creation of a network of relations. Research has established an association between social integration and health (e.g. Kawachi \& Berkman, 2000). Fleuret \& Atkinson (2007) articulate a relationship between positive wellbeing and integration of people into "a rich network of social associations" (p. 113). In the writing sessions, the development and continuation of social relations between participants were the primary facets of examination, along with the opportunities these social relations created. 


\section{Space of Security}

Security needs are foundational to wellbeing, according to Maslow (1954). Spaces of security emphasise a sense of 'felt safety' (Purvis, Cross, Dansereau, \& Parris, 2013) that empowers a person to learn and grow and encompasses the relationship between social, spatial, and individual support. In the Write-Ins, we examine the need for security, support, and affirmation to nourish the capacity for embracing the cognitive difficulty that doctoral-level study requires of students.

\section{Therapeutic Spaces}

This dimension recognises the role of social space as a factor which contributes positively to the construction of a therapeutic space (Fleuret \& Atkinson, 2007). A key focus is the realities of spaces for doctoral candidate replenishment, including emotional and cultural aspects. In recognition of the extended time frames of doctoral candidature, this dimension considers spatial qualities that sustain motivation throughout the candidature, for example, psychological capacity among collectives (Dawkins, Martin, Scott, \& Sanderson, 2015).

SoWT was chosen over the other forms of possible conceptual frames for analysis because of its specific emphasis on wellbeing. We were interested in learning about how the spatial qualities of the writing group impacted upon our wellbeing as doctoral candidates, to inform future approaches to supporting student wellbeing in higher education.

\section{Method}

We seek to situate our research and program evaluation within its social, cultural, economic, and political contexts (Kincheloe, 2005). The research is positioned within 
an interpretive paradigm (Smith, 2008) and emphasises understanding human experience by investigating its underlying meanings, purposes and interpretations (Morehouse, 2012). We embrace a collaborative autoethnographic approach that acknowledges researchers as participants in a shared community or with a shared experience (Chang, Ngunjiri, \& Hernandez, 2013). It is a process of writing and thinking about self, collaboratively. According to Chang, Ngunjiri \& Hernandez (2013) collaborative autoethnography allows researchers to 'similarly benefit from self and collective analysis'. The approach allows an in-depth exploration of a phenomena from an insiders experiential perspective, while the 'inclusion of multiple voices and perspectives adds rigor to autobiographic interrogation' (p. 25).

Similar to other scholars, we used collaborative autoethnography to explore our experiences of Write-Ins and how they influenced wellbeing in different ways (HainsWesson \& Young, 2016; Nairn et al., 2015). Here, as a community of researchers, we became the researched. We were able to write and reflect from different vantage points and work iteratively to deepen the reflexive process during data collection by probing into spaces of team members' storying, revealing taken-for-granted events that form critical incidents for exploration (Chang et al., 2013). In addition, we acknowledge the importance of the ethics of our research which falls in the contested realm of collaborative autoethnography (e.g. Chang et al., 2016, Forber-Pratt, 2015; Guyotte \& Sochacka, 2016; Lapadat, 2017) and accordingly we sought advice from our Institutional Chair of Ethics to address ethical considerations. We adopted ethical principles in the conduct of the research through seeking voluntary consent to participate, deidentifying interview transcripts and preserving participant confidentiality by applying gender-neutral pseudonyms. 


\section{Data Generation}

Data generation is borrowed from Chang, Ngunjiri, and Hernandez's (2013) concurrent autoethnographic process, which sees the authoring team become 'the researched'. All authors participated in generating written reflections and interviews. At the time of participating in the Write-Ins, we were full-time $\mathrm{PhD}$ candidates, six enrolled in third year, two in their second year, and one in first year of their candidature. Two of us identify as male and seven as female. We were each invited to reflect on our engagement with the program, and the impact this had on our wellbeing. Reflections were captured in semi-structured interviews conducted immediately after a writing session. Interviews are a valuable way of profoundly understanding insights, particularly in program evaluation. Use of interviews was essential to ensure that we all had equal opportunity to provide our perspectives (Clarke, 2006). Interviews were transcribed verbatim, rather than by summary, to enable accurate future coding. Importantly, as the researchers were also the researched, we adopted interviews as a way of exploring our thoughts with reduced self-bias, while still upholding a participatory design. These interviews were framed by asking the following three questions.

(a) What was your experience of the Write-In sessions?

(b) How have the sessions influenced your wellbeing?

(c) Can you reflect on the practicalities (e.g. facilities, catering) of the sessions? We recognise reflective writing is an increasingly valued method and core to collaborative autoethnography (Van Herk, Smith \& Gold, 2012). As such we were all given the opportunity to respond to the same three interview questions in written form 
(ranging from 300-1,000 words in length) in addition to participating in an interview. A total of seven written reflections were generated through this process.

\section{Analysis}

Spaces of wellbeing theory was employed as a coding frame for the analysis. Four independent researchers simultaneously analysed the data from one of the four dimensions of SoWT (therapeutic spaces, integrative spaces, spaces of security, and spaces of capability) (Fereday \& Muir-Cochrane, 2006). This hybrid method of coding began with a deductive approach, using SoWT dimensions from the data. Following Fereday and Muir-Cochrane (2006), the stages of code reliability, integration, connection, and legitimisation occurred collaboratively among the team through collaborative research team discussions held during processes of analysis. Autoethnographic processes are sometimes critiqued for being self-indulgent and lacking rigour (Lapadat, 2017). We adopt the assumptions of Delamont (2009), who argues in favour of ethnographers reflexively seeking to understand research-related interpersonal dilemmas.

In some instances, data were coded to more than one dimension of the SoWT theory and we acknowledge some blurring of boundaries between the dimensions of the SoWT framework. Nevertheless, we contend that the framework is suitable for the analysis of the study because of the explicit focus on ways that space constitutes and contribute to wellbeing and the importance of the issue of doctoral student wellbeing which was the focus of the analysis. Areas of overlap provided opportunities to consider and discuss collaboratively the multiple ways that some aspects of a Write-In work to support candidates. For example, a collaborative space could work to support wellbeing for more than one reason by providing mental respite [therapeutic space], opportunities 
for social interaction [integrative space] and opportunities to talk through particular struggles of $\mathrm{PhD}$ work [spaces of capability].

\section{Findings}

The following sections report the findings of the study, corresponding with the SoWT dimensions (Fleuret \& Atkinson, 2007), before highlighting the interdependency and opportunity for the synthesis between these discrete spaces in the discussion.

\section{Spaces of Capability}

Through the Write-Ins, participants generated text for their thesis and described the conditions within these sessions as helping to make possible the production of this thesis writing. In this sense, the Write-Ins were a space of capability that extended candidates' capacity to produce the work most pressing to them. Participants often recited word counts achieved, chapter completions and readings in their responses. For example, in a written reflection, Jordan commented 'I was able to draft and then re-draft a chapter (around 4k)', Jamie noted 'I have written about 2,000 words and read about 10 articles', and Leigh said 'I've written about 5000 words, on top of reading and notetaking'. After attending one session, Darcy wrote in a written reflection 'I managed to write probably 1,000 words for the draft of a paper'. The production of the thesis was a common purpose among participants and formed a compelling collective value for the group.

Conducting the writing sessions on a weekend provided students with a day outside of their usual schedules to concentrate on their doctoral work. Similar to recent studies (Crawford \& Probert, 2017), many of the participants were balancing family, 
life, and work commitments while completing their doctorate. For example, in an interview, Frankie said:

It's weekends like this where I do have that time to actually maximize my thesis writing..... I can just concentrate on my [thesis] work. I'm not teaching, I'm not trying to write and work while he [my son] is at school for five or six hours a day.

This time of concentration and little distraction was noted as a critical attribute to ensuring productivity in the sessions (comments included e.g. 'mostly for focusing and not having any distractions' [Bailey, interview] and 'In a day of concentrated attention, it was possible to complete more than what I would typically undertake in a week' [Jordan, interview]). While there are many writing and research scholars offering advice for doctoral candidates on time management and managing procrastination, the Write-In sessions offered an opportunity for time-pressured candidates to find focused writing time with peer support (Gardiner \& Kearns, 2011).

\section{Integrative Spaces}

Participants suggested that the socio-spatial relations of the Write-In sessions contributed positively to their well-being. Working in a group afforded respite away from the isolation that was seen as a normal part of the doctoral experience by participants. For example, Frankie, stated in an interview, 'I often do feel isolated in my situation, because I am homebound and I work from home, and it's a tumultuous environment to work in, so this is actually really, really, nice.' Data showed that three distinct integrative spaces were created during the course of the Write-In, each with a dedicated purpose and spatial boundary. These spaces were workspaces, collaborative spaces, and social spaces. 


\section{Work Space}

The main space of capability was regulated as a quiet space set around a communal board table. Both physical and intangible attributes of the work space contributed to participants experiences.

Participants outlined vital physical attributes of the work space, with the use of a communal table rather than individual stations as a motivator, while others noted the small scale of the room in relation to the group size: Jesse indicated the importance of all being 'disconnected from where we normally work and we're together here, in one place to do what we do together' (interview). The furniture arrangement also impacted the establishment of comradery in the work-space as Jesse goes on to say: 'When you're all together and you're looking at other people... The way that people behave is when they see other people doing something, it helps them to motivate them to do similar things'.

\section{Collaborative Space}

In addition to the work space, a separate but adjacent break-out space was available. The socio-spatial relations that defined this collaborative space enabled peer-to-peer learning and ideas sharing. The break-out space (couches, chairs, and tea and coffee facilities) was freely available during working time to offer a space of collaboration. Some participants highlighted the sharing of ideas and working methods as a distinct feature of the collaborative space, in that it enabled 'sharing ideas/methods across different disciplines' (Bailey). Jordan (interview) highlighted the importance of being able to seek contact with others during designated Write-In time, where 'you can have a cup of tea and a little bit of a debrief with somebody out in a tearoom and then come back and get your head back in the game'. 


\section{Social Space}

The incorporation of an externally-funded group lunch allowed for the establishment of a dedicated and relaxed social space. This space enabled an effective replenishment of psychological wellbeing that enhanced productivity during other times (Luthans, Youssef \& Rawski, 2011). As Frankie recalled, 'I think it was really nice to have that break at 12 o'clock to go and have some lunch... it's nice to have that bit of outlet and have a bit of chit-chat then settle down and get back into work again.' (interview), 'You come back and you're not lulling about... You can be really focused when you are there' (Jordan, interview).

The social space was defined by the opportunity to network across disciplines in a low-pressure environment. The Write-Ins were also found to be integral to the sharing of experience. Participants benefited from sharing discipline-specific procedures and experiences with others from diverse fields. Participants came to recognise that some experiences were common to the postgraduate experience: Common concerns deliberated over during lunch included methodology, procedure, and quality of supervision.

\section{Space of Security}

Participants noted the benefit they experienced from sharing their doctoral experiences and hearing others' experiences. This form of collegial support afforded participants opportunities to learn from their peers, about how to navigate ill-defined problems of candidature and to make sense of their own identities as doctoral candidates. For example,

Most of my week, I am working in a solitary way, so it makes a massive difference to share this journey, and to hear other people's experiences, and that's levelling 
and reassuring and encouraging and productive for my own progress. (Charlie, interview)

Participants highlighted the sense of belonging and reduction in their feelings of social isolation which developed through their involvement with the Write-In group. For example, 'I feel like I'm part of the group, where often I don't feel like I'm part of the group, because I'm always separated from everybody...like other HDR students, I feel like I'm doing this all on my own' (Frankie interview). The Write-Ins importantly provided candidates with opportunities to talk through difficulties they encountered in their writing and candidature more broadly during the lunch breaks and in other incidental conversations on the Write-In days. Being together writing, sharing, and learning together engendered security in the participants:

You go through experiences... many are key milestones. You get [external] feedback but [here] you've got someone else to talk about ideas...like "this has happened with me", then that shit will happen with them. It helps you to triangulate what's happening in your doctorate with what's happening with other [candidates]. (Jesse, interview)

Findings suggest that the way the Write-Ins were structured enabled the formation of a space that was supportive of candidates' ontological security. This security was supported through candidates' sharing of experiences that help in the setting of expectations, resonating with Mitzen's (2006) contention that:

Individuals need to feel secure in who they are, as identities or selves. Some deep forms of uncertainty threaten this identity security. The reason is that agency requires a stable cognitive environment. Where an actor has no idea what to expect, she cannot systematically relate ends to means, and it becomes unclear how to pursue her ends. (p. 342). 
Ontological security is the feeling of security in the inferred inner self and moral self, not the body self (Epstein, 1973). The peer conversations that took place on WriteIn days were opportunities for critique and self-reflection on writing, which provided space for candidates to make sense of their experiences through and with others. The normalisation of candidature experiences contributed to a stabilising 'cognitive environment' which helped candidates to propel their writing forward (Mitzen, 2006, p. $342)$.

\section{Therapeutic Spaces}

Participants suggested that attending the writing groups directly reduced the stress and anxiety they experienced related to completing their doctoral studies. For example, Darcy stated during interview, 'I find that having come along here the times that I have and made the progress that I have has worked to alleviate some of that pressure and some of that stress'. Doctoral candidates often place themselves under considerable pressure and express feeling as though they are not reading enough, writing enough, or spending enough time on the thesis (Bazrafkan et al., 2016). Yet participants gained satisfaction from feeling as though they had made progress: 'Just that knowing of having done something, makes me feel a whole lot better' (Jamie, interview). The Write-In sessions also provided a haven away from the self-imposed unhealthy work practices that occurred during the week. For example, 'You don't have to feel guilty for all the things that you haven't done while you're having lunch, which seems to be my experience during the week' (Jamie, interview). Stress and anxiety can be crippling for productivity and impact negatively on wellbeing (Bazrafkan et al., 2016; Cotterall, 2013). 
The Write-In sessions offered a positive response to these negative emotions and acted to facilitate participants experiencing positive wellbeing. Participants reported the sharing of space with other candidates who were working towards similar goals and had similar experiences as contributing positively to their wellbeing. For example, 'I enjoyed being able to spend time with people who are motivated to finish - ie. Those at our late stage of candidature. It was a strong motivator for me' (Jesse, interview) and:

I find that there's something really valuable about being in a room full of people who are here for a purpose and everybody's here for essentially the same purpose. There's something about that that drives you or motivates you to continue doing work. (Bailey, interview)

The motivation of a group of doctoral candidates working towards the common goal of finishing their thesis helped to create a space of capability with therapeutic benefits for candidates. Together, these session attributes of common-stage goals and shared appreciation of group working were found to be therapeutic for participants dealing with the many challenges of their doctoral studies. While these therapeutic benefits positively contributed towards doctoral student wellbeing, we contend that it was through the complex interconnections between the various spaces created through the Write-Ins which worked to support doctoral candidates' progress.

\section{Discussion}

The Write-Ins supported candidates in numerous ways; they were not just about writing, nor were they focused directly on socialising. The Write-Ins provided opportunities for participants to work independently towards self-identified goals, alongside others with a shared experience (Cuthbert, Spark and Burke, 2009). The sessions were therapeutic spaces and contributed to doctoral candidates feeling better about their studies (Wilmot 
$\&$ McKenna, 2018). The physicality of space contributed to the effectiveness of the Write-Ins, but so too were the temporal and social components of space (Maher et al., 2008). The Write-Ins carved out an expanse of time that was devoid of the typical interruptions of the working week (including personal responsibilities and commitments) and provided doctoral candidates with the mental space for thesis writing, also found essential to candidates in other studies (Crawford \& Probert, 2017; Wilmot \& McKenna, 2018).

For participants, the Write-Ins offered a space of capability to complete writing tasks. After attending the Write-Ins, doctoral candidates could look back and see their written progress. The act of producing written words worked to alleviate stress and anxiety. Through this space of capability, most candidates responded to the question of what they achieved during the sessions by highlighting, often proudly, a word-count. This indicates that the number of words written during a session was the most common and precise evaluative mechanism of individual success.

Writing a thesis is a high-stakes productive output, and the anxiety involved in the production (along with the risk of failure) can itself be a barrier to some candidates being able to write. Research demonstrates that, for many doctoral students, the heightened stress and anxiety experienced during their period of candidature is, in part, attributable to continuous critique of the candidate's doctoral writing (Francis, Mills, Chapman, \& Birks, 2009). This critique can be viewed as a continuous unlearning (or fracturing) of beliefs or ideas which candidates may formerly have held, and thus be experienced as a threat to their ontological security (Mitzen, 2006), which a space of security can respond to.

Consistent with Verlie et al. (2017), the conditions within the Write-Ins were found to contrast with this expected mode of critique through the creation of a 
supportive and comfortable environment, reducing the sense of anxiety around writing. Simple strategies that contributed to this reduction of anxiety were the use of a communal space where participants could see others in similar positions as them (e.g. shared hardship), the use of an unfamiliar space offering a 'shock to the system', and the close proximity between but separation of spaces of integration (break-out room) and spaces of capability (workroom). These conditions enabled candidates to write continuously, and progress closer to the completion of their doctoral program and offered a space of liminality (Atkinson \& Robson, 2012). Although the participants expressed appreciation for the individual elements of the Write-Ins (e.g. as spaces of capability, spaces of security, therapeutic and integrative spaces), it was often the synthesis of these elements that were perceived to be most beneficial to the candidates' wellbeing.

\section{Surviving in the Neoliberal Academy Sees Candidates Doing it for Themselves}

Throughout this paper, we have noted the impact that neoliberalisation of universities is having on candidate wellbeing, yet, we acknowledge that the Write-Ins too were a product of the neoliberal regime. Doctoral study in Australia, as in other post-industrial 'late modern' nations, occurs within a neoliberal landscape which places value on economic imperatives and continually works to create efficiencies in modes of production (such as 'producing' a doctoral completion) (Martin, 2016). According to Harvey (2005), under this regime, the economic return of the institution is perceived as the priority rather than the wellbeing of the individual $\mathrm{PhD}$ candidate. The weekend Write-In sessions are representative of the entrepreneurial quality valued and promoted the individual within neoliberal ideology. They represent a "responsibilising" of individuals for systemic problems (Schwanen \& Atkinson, 2015), and seemingly release 
the institution from addressing the structures that create the conditions necessitating such initiatives in the first place. We acknowledge the problematic nature of suggesting that weekend Write-Ins should compensate for insufficient, appropriate writing support mechanisms offered by the university and argue that the supports which help candidates to engage in productive writing sessions should be a priority for universities to provision during weekday programming.

Therefore, while we commend the student union that supported candidates in this study, and candidates creating the support they require themselves, we argue that providing candidate support is core university work. Through researching small scale initiatives such as this, we seek to extend the evidence base for initiatives such as WriteIns, providing evidence for candidates lobbying for further support from their institutions.

\section{Conclusion}

In this paper, we considered how self-organised Write-In sessions became a valued space of wellbeing for doctoral candidates. We extend upon Fleuret and Atkinson's (2007) spaces of wellbeing theory to highlight the overlap between each of the four spaces, as well as how they impacted candidates' wellbeing. The Write-Ins generated capability for candidates through enabling thesis production, social interaction, mental space, and flexible working arrangements and provided some insight into practice-based solutions to address the "mental health crisis in graduate education" noted by Evans et al. (2018, p. 282). This study also reveals how a sense of comradery developed between a group of doctoral candidates from diverse disciplinary backgrounds through the sharing of a more substantial experience of undertaking a Doctorate, challenging initiatives that maintain disciplinary silos. 
Our analysis suggests that institutional culture contours the conditions of possibility for postgraduate students in complex ways that inevitably impact on the person. The weekend Write-In program was a response to the neoliberal conditions of candidature. For example, we suggest that participating in practices that erode candidates' weekends which are assumed for leisure and rest (at least historically recognised as the time for leisure and rest) may further exacerbate the institutional cultures that lead to ill-being. Yet candidates in this study were willing to take part in weekend Write-Ins which suggests that at least for some candidates, candidature is not separated from everyday lives (ie. something that can be 'clocked off' from at 5pm), but rather, interwoven through everyday life, due to the scope of the undertaking that is the $\mathrm{PhD}$ in contemporary neoliberal conditions.

While we acknowledge that the small sample size in this study is a limitation, we consider that our work affirms the importance of wellbeing embedded into thesis producing initiatives, such as writing groups, and that it offers insights into the attributes of initiatives that may work for others and the well-established need for greater institutional support. We recognise that this paper focuses on our experiences of self-initiated writing sessions and acknowledge that Write-Ins may be experienced differently by other candidates. Culture, beliefs and perspectives, and lived experiences colour how events are experienced and understood by individuals (Smith, 2008). What we offer in this paper, however, is a consideration of one strategy for improving doctoral candidate wellbeing. It is opportune for further research to explore the findings generated from this small study and to replicate similar initiatives in other contexts to validate our conclusions about the effectiveness of Write-In-style writing sessions for enhancing doctoral candidate wellbeing. 


\section{Acknowledgements}

The authors would like to acknowledge the funding support for the Write-Ins that was provided by the university's student union. 


\section{References}

Apple, M. (2009). Global crises, social justice, and education. New York, NY: Routledge.

Atkinson, S., \& Robson, M. (2012). Arts and health as a practice of liminality: Managing the spaces of transformation for social and emotional wellbeing with primary school children. Health \& Place, 18(6), 1348-1355.

Barry, K., Woods, M., Warnecke, E., Stirling, C., \& Martin, A. (2018). Psychological health of doctoral candidates, study-related challenges and perceived performance. Higher Education Research and Development, 37(3): 468-483, DOI: 10.1080/07294360.2018.1425979.

Baylis, J., Smith, S., \& Owens, P. (2017). The globalization of world politics: An introduction to international relations. Oxford, UK: Oxford University Press.

Bazrafkan, L., Shokrpour, N., Yousefi, A., \& Yamani, N. (2016). Management of stress and anxiety among PhD students during thesis writing: A qualitative study. Health Care Manager, 35(3), 231-240.

Chang, H., Ngunjiri, F., \& Hernandez, K. (2013). Collaborative autoethnography. Walnut Creek, CA: Left Coast Press.

Chang, H., Ngunjiri, F., \& Hernandez, K. (2016). Collaborative autoethnography. New York, NY: Routledge.

Clarke, A. (2006). Qualitative interviewing: Encountering ethical issues and challenges. Nurse Researcher, 13(4), 19-29.

Cotterall, S. (2013). More than just a brain: emotions and the doctoral experience. Higher Education Research \& Development, 32(2), 174-187.

Crawford, J., \& Probert, D. (2017). 2017 Postgraduate Experience Survey. Tasmanian University Union Postgraduate Council.

Cuthbert, D., Spark, C., \& Burke, E. (2009). Disciplining writing: The case for multidisciplinary writing groups to support writing for publication by higher degree by research candidates in the humanities, arts and social sciences. Higher Education Research \& Development, 28(2), 137-149.

Dawkins, S., Martin, A., Scott, J., \& Sanderson, K. (2015). Advancing conceptualization and measurement of psychological capital as a collective construct. Human Relations, 68(6), 925-949.

Delamont, S. (2009). The only honest thing: Autoethnography, reflexivity and small crises in fieldwork. Ethnography and Education, 4, 51-63. 
Diener, E., \& Lucas, R. (2000). Explaining differences in societal levels of happiness: Relative standards, need fulfilment, culture, and evaluation theory. Journal of Happiness Studies, 1(1), 41-78.

Emery, S., Beasy, K., Crawford, J., \& Green, W. (2018). Drowning in the shallows: an Australian study of the PhD experience. ECER 2018 Abstracts, 4-7 September 2018, Bolzano, Italy.

Epstein, S. (1973). The self-concept revisited: Or a theory of a theory. American Psychologist, 28(5), 404-416.

Evans, T., Bira, L., Gastelum, J., Weiss, L., \& Vanderford, N. (2018). Evidence for a mental health crisis in graduate education. Nature Biotechnology, 36(3), 282-284.

Fereday, J., \& Muir-Cochrane, E. (2006). Demonstrating rigor using thematic analysis: A hybrid approach of inductive and deductive coding and theme development. International Journal of Qualitative Methods, 5(1), 80-92.

Fleuret, S., \& Atkinson, S. (2007). Wellbeing, health and geography: a critical review and research agenda. New Zealand Geographer, 63(2), 106-118.

Forber-Pratt, A. (2015). 'You're going to do what?' Challenges of autoethnography in the academy. Qualitative Inquiry, 21(9), 821-835.

Francis, K., Mills, J., Chapman, Y., \& Birks, M. (2009). Doctoral dissertations by publication: Building scholarly capacity whilst advancing new knowledge in the discipline of nursing. International Journal of Doctoral Studies, 4, 97-106.

Gardiner, M., \& Kearns, H. (2011). Turbocharge your writing today. Nature, 475, 129130.

Guyotte, K., \& Sochacka, N. (2016). Is this research? Productive tensions in living the (collaborative) autoethnographic process. International Journal of Qualitative Methods, 15(1), 1-11.

Hains-Wesson, R., \& Young, K. (2017). A collaborative autoethnography study to inform the teaching of reflective practice in STEM. Higher Education Research \& Development, 36(2), 297-310.

Harvey, D. (2005). A brief history of neoliberalism. Oxford, UK: Oxford University Press.

Higher Education Standards Panel [HES]. (2017). Final Report - Improving retention, completion and success in higher education. Canberra, Australia: Australian Government Department of Education and Training.

Kawachi, I., \& Berkman, L. (2000). Social cohesion, social capital, and health. In L. F. Berkman, \& I. Kawachi (eds.), Social epidemiology (pp. 174-190). New York, NY: Oxford University Press. 
Kilpatrick, S., Emery, S., Adler, V., \& Farmer, J. (2019). Social enterprises and wellbeing for disadvantaged people in Australian regional towns. Paper presented at National Rural Health Conference: Better Together, Hobart. http://www.ruralhealth.org.au/15nrhc/sites/default/files/A10-2_Kilpatrick.pdf

Kincheloe, J. (2005). On to the next level: Continuing the conceptualization of the bricolage. Qualitative Inquiry, 11(3), 323-350.

Lapadat, J. (2017). Ethics in autoethnography and collaborative autoethnography. Qualitative Inquiry, 23(8), 589-603.

Luthans, F., Youssef, C., \& Rawski, S. (2011). A tale of two paradigms: The impact of psychological capital and reinforcing feedback on problem solving and innovation. Journal of Organizational Behavior Management, 31(4), 333-350.

Maher, D., Seaton, L., Mcmullen, C., Fitzgerald, T., Otsuji, E., \& Lee, A. (2008). 'Becoming and being writers': The experiences of doctoral students in writing groups. Studies in Continuing Education, 30(3), 263-275.

Martin, C. (2016). The sharing economy: A pathway to sustainability or a nightmarish form of neoliberal capitalism?. Ecological Economics, 121, 149-159.

Maslow, A. (1954). Motivation and personality. New York, NY: Harper \& Row.

Mewburn, I., Osborne, L., \& Caldwell, G. (2014). Shut up \& Write! In C. Aitchison \& C. Guerin (eds.), Writing groups for doctoral education and beyond: Innovations in practice and theory. Abingdon, UK: Routledge.

Mitzen, J. (2006). Ontological security in world politics: State identity and the security dilemma. European Journal of International Relations, 12(3), 341-370

Morehouse, R. (2012). Beginning interpretative inquiry: A step-by-step approach to research and evaluation. Florence, KY: Routledge.

Nairn, K., Cameron, J., Anakin, M., Juntrasook, A., Wass, R., Sligo, J., \& Morrison, C. (2015). Negotiating the challenge of collaborative writing: Learning from one writing group's mutiny. Higher Education Research \& Development, 34(3), 596608.

Nussbaum, M. (2001). Women and human development: The capabilities approach. Cambridge, UK: Cambridge University Press.

Purvis, K., Cross, D., Dansereau, D., \& Parris, S. (2013). Trust-based relational intervention (TBRI): A systemic approach to complex developmental trauma. Child \& Youth Services, 34(4), 360-386.

Rhoades, G., \& Slaughter, S. (1997). Academic capitalism, managed professionals, and supply-side higher education. Social Text, 51(1), 9-38. 
Sampson, K., \& Comer, K. (2010). When the governmental tail wags the disciplinary dog: Some consequences of national funding policy on doctoral research in New Zealand. Higher Education Research \& Development, 29(3), 275-289.

Schwanen, T., \& Atkinson, S. (2015). Geographies of wellbeing: an introduction. Geographical Journal, 181(2), 98-101.

Schyns, P. (1998). Crossnational differences in happiness: Economic and cultural factors explored. Social Indicators Research, 43, 3-26.

Silverman, M., Meyer, P., Sloane, F., Raffel, M., \& Pratt, D. (1997). The big ten student suicide study: A 10-year study of suicides on midwestern university campuses. Suicide and Life-Threatening Behavior, 27(3), 285-303.

Smith, J. (2008). Interpretive Inquiry. In L. Given (eds.), The SAGE encyclopedia of qualitative research methods. Thousand Oaks, CA: SAGE Publications.

Tan, A., Lamontagne, A., Sarmugam, R., \& Howard, P. (2013). A cluster-randomised, controlled trial to assess the impact of a workplace osteoporosis prevention intervention on the dietary and physical activity behaviours of working women: Study protocol. BMC Public Health, 13, 405.

Tertiary Education Quality and Standards Agency. (2018). Higher education standards framework guidance note: Wellbeing and safety. Canberra, Australia: Australian Federal Government.

Van Herk, K., Smith, D., \& Gold, S. (2012). Safe care spaces and places: Exploring urban Aboriginal families' access to preventive care. Health \& Place, 18, 649656.

Verlie, B., Emery S., Osborn, M., Beasy, K., Coleman, B., Kezabu, K. \& Nicholls, J. (2017). Becoming researchers: Making academic kin in the Chthulucene. Australian Journal of Environmental Education, 33(3), 145-159.

Waight, E., \& Giordano, A. (2018). Doctoral students' access to non-academic support for mental health. Journal of Higher Education Policy and Management, 40(4), $390-412$.

Wheatley, D. (2017). Autonomy in paid work and employee subjective well-being. Work and Occupations, 44(3), 296-328.

Wilmot, K., \& McKenna, S. (2018). Writing groups as transformative spaces. Higher Education Research \& Development, 37(4), 868-882.

Woodgate, R., Zurba, M., Edwards, M., Ripat, J., \& Rempel, G. (2017). The embodied spaces of children with complex care needs: Effects on the social realities and power negotiations of families. Health \& Place, 46, 6-12 